\title{
Disclosure of Diagnosis and Prognosis to Cancer Patients in Traditional Societies: A Qualitative Assessment from Lebanon
}

\author{
James Feghali'), Hussein El Semesmani'), Abdallah Abboud'), \\ Lily Chacra'), Khalil Choucair'1), Madelin Fenianos'), \\ Lama Al Jebbawi'), Farah El Sayed '), Faysal Kak²), Salim M. Adib²) \\ 1)Faculty of Medicine, American University of Beirut (AUB), Lebanon \\ 2)Faculty of Health Sciences, American University of Beirut (AUB), Lebanon
}

\begin{abstract}
Background: The issue of when, how, and whether to disclose full information about cancer diagnosis and prognosis to patients is still debated in some parts of the world, including Lebanon. Despite formal academic emphasis on a larger autonomy for Lebanese patients in deciding the course of their disease, there has been no apparent impact on either clinical practices nor public expectations. The topic of full disclosure is rarely if ever discussed in open fora, or in mass media channels in Lebanon.

Subjects and Method: Seven key stakeholders were identified and interviewed regarding obstacles to spelling out clear guidelines within our national context. The interviews were transcribed and subsequently analyzed for recurrent patterns and concepts.

Results: Senior oncologists interviewed generally favored gradual disclosure and most perceived a changing trend among both patients and physicians towards more disclosure. They also agreed on a need for the formal training of residents and fellows to better communicate bad news to patients. All the interviewed physicians attested to the benefits of candid disclosure in terms of patient psychology and overall wellbeing. They also mentioned that psychological services, which may facilitate the disclosure process, are greatly under-utilized in oncology. Lawyers highlighted the vagueness of the current Lebanese legislation regarding the obligation of truthful disclosure in comparison to laws in developed countries and the implications on patient autonomy.

Conclusion: The study identified the need for improvements at various levels, including interventions to modify the expectations of the Lebanese public regarding cancer disclosure and to clarify existing legislative texts.
\end{abstract}

Key-words: Ethics; Legislation; Middle-East; Disclosure

\section{Correspondence:}

James Feghali. Faculty of Medicine, American University of Beirut (AUB), Lebanon, 1101 North Calvert Street, 610, Baltimore, Maryland, 21202. E-mail: jfeghal2@jhmi.edu.

Telephone: +1-(267)-595-9995.

\section{BACKGROUND}

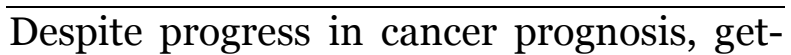
ting a full diagnosis still overwhelms patients and family members both physically and mentally (Kazdaglis et al., 2010; Dégi, 2009). Different countries approach disclosure differently (Mystakidou et al., 2004). Currently, acknowledgment of the disease is the norm in many developed countries including most of Europe and North America. In such regions, patient autonomy takes precedence, and laws support the moral obligation of truth telling (Wood et al., 2009). On the other hand, the issue of when, how, and whether to disclose full or partial information about diagnosis and prognosis to cancer patients is still disputed in some traditional countries such as Japan, Greece, Italy, Turkey, and Lebanon (Elwyn et al., 2002; Tsoussis et al., 2013; Grassi et al., 2000; Oksüzoğlu et al., 2006; Naji et al., 2015). In the Middle Eastern 
Journal of Epidemiology and Public Health (2018), 4(2): 109-116

https://doi.org/10.26911/jepublichealth.2019.04.02.06

culture, families play a large intermediate role in receiving the information, translating it to the patient, and discussing therapeutic options with the providers (Khalil, 2013). Their views are "strongly tied to social norms and traditions" that resist the "western conception" of full and direct disclosure (Surbone, 2006).

The issue of disclosure has been considered from several angles in Lebanon since the 1990 s. In a study conducted in 1998 among physicians likely to encounter cancer cases in their practice, $47 \%$ usually disclosed the truth to cancer patients. The tendency to disclose was strongly associated with older practitioners with lengthier clinical experience and with providers of specialized surgical or medical care rather than primary health care (Hamadeh and Adib, 1998). A more recent study in 2015 showed that not much had changed in the preference for consistent disclosure in almost two decades. In that paper, " $83 \%$ of physicians in Lebanon preferred disclosing diagnosis and prognosis to their patients. However, only $40 \%$ revealed the true diagnosis to the patients themselves and just 9\% disclosed the information immediately after the diagnosis is known" (Farhat et al., 2015).

Concerning preferences in the public, a 1999 study showed that $42 \%$ of respondents preferred not having the truth about serious diseases fully disclosed to patients (Adib and Hamadeh, 1999). This study predicted that as the Lebanese public's level of awareness about the effectiveness of medicine increases, the expectations for concealment will decrease (Adib and Hamadeh, 1999). However, the expectations may be different when cancer becomes a reality and not just a hypothetical possibility. In $2015,80 \%$ of relatives of cancer patients in Lebanon declared not being in favor of revealing detailed information about the disease to the patient directly. Physicians, being aware of this, tend to inform the family first about a cancer diagnosis, seeking their approval regarding the degree of information to be provided to the patient (Farhat et al., 2015).

The Lebanese legislation upholds the right of patients to be completely informed about their diagnosis, the proposed management plan, the existence of therapeutic options, and the disease prognosis. It also binds the doctor to disclose to patients all the information concerning their disease. However, if the patient does not wish to be informed, then these wishes must be respected. Under such circumstances, the patient can appoint a representative to be informed of the condition and treatment plan. In addition, if it were established that the disclosure of the diagnosis would carry a negative impact on the patient and the course of treatment, then the doctor can exceptionally reveal the information gradually for the patient's benefit (Law 574, 2004).

Despite formal academic emphasis on a larger autonomy for Lebanese patients in deciding the course of their disease, there has been no apparent impact on neither clinical practices nor public expectations. The topic of full disclosure is rarely if ever discussed in open fora, or in mass media channels in Lebanon. In this qualitative paper, we have interviewed several medical and non-medical stakeholders to obtain their views regarding the changing patterns of disclosure behavior or the absence thereof in Lebanon as we approach 2020.

\footnotetext{
SUBJECTS AND METHOD

\section{Study design}

Domains of factors influencing disclosure of diagnosis/prognosis to cancer patients were identified based on a thorough literature review and after consulting with medi-
} 
cal experts. These were: physician-patient interaction, psychological support in disclosure, and legislation regarding medical conduct. Key stakeholders with expert knowledge in any one of those domains were determined and interviewed.

\section{Sources of data}

Seven key stakeholders were interviewed. They included three foreign-trained oncologists working at the American University of Beirut Medical Center (AUBMC) and one working at the Military Hospital in Beirut. One interview was conducted with a foreign-trained psychiatrist working at AUBMC. A lawyer with training in international law currently practicing in and around the capital city Beirut was identified to discuss legal aspects of the issue. Finally, an interview was conducted with a Member of Parliament (MP) who had been serving as Chairman of the Health Committee for more than 15 years. Given that the vast majority of cancer patients in Lebanon ended up being treated in the Greater Beirut area, the stakeholders selected offered a representative view of cancer disclosure practice in Lebanon.

\section{Procedures}

All interviews were scheduled in the fall of 2017 via email, with an explanation of the purpose and structure of the encounter. They were conducted face-to-face by members of the research team. A semi-structured format was used, which included the same set of core open questions and other variable ones tailored to the domain of expertise of each stakeholder. The interviews were transcribed and subsequently analyzed for recurrent patterns and concepts.

\section{RESULTS \\ 1. Aspects of physician-patient inter- action affecting disclosure}

These aspects include the physicians' current practice of disclosure, perceived trends in disclosure in Lebanon, and the effect of their training as medical residents/fellows on their preferred attitude.

Based on personal experience, all the oncologists interviewed were proponents of open disclosure, when possible. Most would routinely ask the patients, prior to any invasive investigation, how much they would like to know about a suspected cancer. All concurred that the method of disclosure is of prime importance. In general, they would favor a gradual disclosure of the severity of the disease that takes place over several visits. One physician regretted that some oncologists rush through the disclosure process before a firm relationship has been established with a patient. The wording used for the disclosure was also a key point emphasized by most interviewees. One oncologist mentioned: "At times I do not use the word cancer, but try to deliver the news using different terms such as "severe inflammation" instead of cancer and "IV medication" instead of chemotherapy". Another mentioned that when cancer is described as a "chronic" rather than a "terminal" illness, the patient accepts the diagnosis more readily.

Three of the interviewed oncologists mentioned that in their experience, trends in cancer disclosure seem to be changing. They perceived that patients were gradually shifting towards more demand for autonomy and more desire for full knowledge. They noticed that this was a salient feature particularly in younger patients. The three oncologists practicing at AUBMC mentioned that disclosure is becoming the norm in their practices, while one oncologist working in another non-University affiliated 
Journal of Epidemiology and Public Health (2018), 4(2): 109-116

https://doi.org/10.26911/jepublichealth.2019.04.02.06

facility, regretted that he did not perceive such trends towards truthful disclosure occurring around him.

All the oncologists reported that they had received no formal training in communication skills needed for breaking bad news during their medical education or training in Lebanon. They confirmed that such training would have facilitated their choice for routine disclosure. Although the foreign-trained oncologists mentioned that disclosure was mandatory in their training, they admitted that they did not always transmit the line of conduct recommended elsewhere to their Lebanese trainees due to the "peculiarities of the region". Only one of the oncologists seemed to have adopted a systematic pattern for breaking bad news, when he deemed it possible or desirable. All physicians interviewed, including the psychiatrist visited for the purposes of this research, highlighted the importance of integrating a formal communication skills course specific for breaking cancer news in training curricula in the Middle-East. Other than disclosing cancer diagnosis, physicians mentioned training to approach all potentially heavy issues such as the discussion of the "do not resuscitate/do not intubate" option and preferences for palliative/end-of-life care.

\section{Psychological support in disclosure}

The oncologists were asked about the psychological dimensions of full outcome disclosure and the utility of psychological services. All the interviewed oncologists attested to the benefits of candid disclosure on patients' psychological wellbeing in their practice. They mentioned that patients who receive full disclosure become more cooperative and receptive to the physician's options for care. They also noticed that the elimination of uncertainty alleviates some of the patients' fear and allows them to better cope with their symptoms. They noted that distrust often arises when disclosure is delayed, which compromises the quality of care delivered.

The psychiatrist believed that unless patients explicitly refuse disclosure, it is always better for their psychological health to know their diagnosis. In addition, he mentioned the importance of using the proper language on patients' psychology. Words that convey empathy and hold less negative connotations can make a big difference in how the news are received: "The more control you have over a situation, the better your state of mind. We can always find a way to deliver the diagnosis in a manner suitable to the patient's level of education and understanding". The psychiatrist mentioned that patients would be undergoing state-of-the art treatment while their mental health needs were being neglected. Depression, anxiety, and PTSD are some of the co-morbidities that might surface with a new cancer diagnosis and later during the long and uncertain treatment process. He indicated that psychological services were greatly under-utilized in oncology in Lebanon, perhaps because oncologists feel they are capable of handling psychological outcomes and prescribing psychotropic medications on their own. Yet, "the oncologist might have a huge patient load and might not give sufficient time for the patient to absorb the news. Here comes the role of the psychological or the palliative care team to intervene and offer support as needed. It is even better for the patient to have a psychologist involved at the time of disclosure." Some oncologists, speaking for the record, emphasized the need for having a psychologist as part of the multidisciplinary team taking care of cancer patients who would "dedicate time to listen to them, comfort them, and assess their psychological state." 


\section{Legislation regarding medical conduct}

All the oncologists interviewed were not aware of any obligation to disclose under the Lebanese law. They could not accurately articulate what was permitted or expected of them in the Lebanese legislation. They all agreed that no institutional policy exists about truthful disclosure of diagnosis/ prognosis to patients at AUBMC or the Military Hospital. The team sought out the AUBMC Administration, which confirmed that this was actually the case.

The research team met the physician serving as Chair of the Health Commission in the Lebanese Parliament. He pointed to the "Right to Medical Care in Lebanon" law 574/2004, which had been enacted and never amended since then. The MP was willing to discuss shortcomings in the law to be considered for eventual modifications. "It is now brought to my attention even more how in practice, disclosing the diagnosis of cancer is not an easy task. It is not easy for the doctor to disclose nor is it easier to conceal information from patients. I think there is a great need for psychological support in determining patient readiness concerning disclosure and offering necessary support during treatment. Maybe this new idea can be formulated and added to the law."

\section{Physician-patient interaction}

The results of our study confirm that key stakeholders do not perceive the current practices as consistent and codified. This is similar to the findings of previous Lebanese studies. Failure to meet patients' expectations can be attributed to several social, legal, and psychological factors.

The use of misleading wordings to describe cancer is alarming. These "incorrect" wordings can be widely misinter- preted and actually impede the optimal management course of a cancer case. This practice is in direct opposition to the concept of informed consent and patient autonomy, setting a fertile ground for distrust to arise in the physician-patient relationship. In India, one study showed that several euphemisms are used to replace the word cancer early on in disclosure including "tumor", "growth", and "lump" (Chittem, 2015). Even such words were construed by some oncologists of the study as a dilution of the message, which in effect constitutes lying (Chittem, 2015).

Considering the difficulty of communicating dismal prognoses to patients, medical guidelines on breaking bad news have been published, such as the SPIKES protocol (Baile et al., 2000; Girgis and Sanson-Fisher, 1995). Several strategies, including didactic lectures and group discussions, have been developed to teach medical students and physicians the methodology of communicating bad news (Rosenbaum et al., 2004). In addition, the incorporation of standard disclosure training into curricula has been shown to be beneficial for trainees (Baile et al., 1999). A survey sent to all the hematology/ oncology fellowship program directors in the US showed that $63 \%$ felt that extensive, formal training is important for skill development in delivering bad news while only $23 \%$ of their fellows actually received considerable training (Hebert et al., 2009). In line with these views, most of the stakeholders in our study see great benefit in formally teaching Lebanese fellows and residents the art of breaking bad news. The Order of Physicians in Lebanon, which holds the ultimate corporate control on medical practice and is consulted regarding all new health-related legislation, should play a leading role with program directors to incorporate a formal 
Journal of Epidemiology and Public Health (2018), 4(2): 109-116

https://doi.org/10.26911/jepublichealth.2019.04.02.06

and structured training program for trainees and attending physicians alike.

\section{Norms and awareness}

Most practitioners attribute the inconsistent practices and tendency to withhold truth to alleged expectations in the Lebanese public. It is very likely that the prevalent traditional culture, which values "keeping hope alive" as a sign of protecting the patient's well-being, will indeed resist full disclosure (Adib, 2004). However, this normative denial attitude is based on obsolete knowledge and awareness regarding cancer prognosis. A 2016 survey of public awareness about malignancy in India, another traditional culture, illustrated that $30 \%$ of responders were unaware that cancer can be curable, that it is not contagious, and that it is not a curse or death sentence (Elangovan et al., 2016). No studies have been done in Lebanon to gauge the public's awareness and perception of cancer in the modern era of personalized therapy, very likely because interventions targeting awareness have not been attempted. As more knowledge is disseminated regarding the treatability and improved outcomes in cancer, expectations should change, prompting modifications in physicians' disclosure practices.

\section{Role of psychology in disclosure}

Psycho-oncology, which emerged about 30 years ago, has "produced a model in which the psychological domain has been integrated, as a subspecialty, into the diseasespecific specialty of oncology" (Holland and Weiss, 2010). The field offers many contributions to training staff in psychological management, taking care of patients and their families, and adding to the existing body of research (Holland and Weiss, 2010). Confirming the benefit of having psycho-oncologists as integrated members of the healthcare team, an Italian study showed that cancer patients receiving chemotherapy with ancillary psychological services had lower anxiety levels, adapted better to their illness, and had higher overall health-related quality of life according to objective self-rating scales (Pugliese et al., 2006). There is ample opportunity to improve the cross-talk between psychologists and Oncology departments in Lebanese hospitals, as there is agreement between stakeholders from both fields that these two disciplines are complementary.

\section{Law}

Stakeholders associated with the legal context spoke about the vagueness of the current Lebanese legislation when it comes to truthful disclosure. What is the timeframe of "gradually" revealing information? How can a physician objectively determine when a diagnosis will carry a stressful toll on the patient? The law reflects great uncertainty as to what constitutes sound medical practice. On the one hand, it mandates truthful disclosure of all information to patients; on the other, it states that certain exceptions can be present without clearly delineating these exceptions. A good benchmark for legal obligations can be seen in the US bill of rights outlining what people being treated for all illnesses should expect to receive from their hospitals, doctors, and nurses. It clearly mentions that "patients should be given complete, accurate information in straight forward language about their diagnosis, prognosis, and treatment plan" (American Hospital Association, 1992). We call upon medical practitioners directly involved with cancer patients to be advocates for rewriting the current law, to reduce the opportunity for subjective interpretation and to enforce the obligation of empowering patients with full knowledge. The issue of disclosure should continue to be monitored as laws and norms change in Lebanon and neighboring countries. 


\section{CONFLICT OF INTEREST}

None of the co-authors has any conflict of interest to disclose.

\section{REFERENCES}

Adib SM (2004). From the biomedical model to the Islamic alternative: a brief overview of medical practices in the contemporary Arab world. Social Science \& Medicine, 58(4), 697-702.

Adib SM, Hamadeh GN (1999). Attitudes of the Lebanese public regarding disclosure of serious illness. Journal of Medical Ethics, 25(5), 399-403.

American Hospital Association (1992). A Patient's Bill of Rights: American Hospital Association catalogue no. 157759. Chicago, IL: The Association.

Baile WF, Buckman R, Lenzi R, Glober G, Beale EA, Kudelka AP (2000). SPIKES-a six-step protocol for delivering bad news: application to the patient with cancer. The Oncologist, 5(4), 302-311.

Baile WF, Kudelka AP, Beale EA, Glober GA, Myers EG, Greisinger AJ, Lenzi R (1999). Communication skills training in oncology. Cancer, 86(5), 887-897.

Chittem M (2015). Responding to family requests for nondisclosure: The impact of oncologists' cultural background. Journal of Cancer Research and Therapeutics, 11(1), 174-180.

Dégi CL (2009). Non-disclosure of cancer diagnosis: an examination of personal, medical, and psychosocial factors. Supportive Care in Cancer, 17(8): 1101-1107.

Elangovan V, Rajaraman S, Basumalik B, Pandian D (2016). Awareness and Perception About Cancer Among the Public in Chennai, India. Journal of Global Oncology, JGO-2016.

Elwyn TS, Fetters MD, Sasaki H, Tsuda T (2002). Responsibility and cancer dis- closure in Japan. Social Science \& Medicine, 54(2), 281-293.

Farhat F, Othman A, El Baba G, Kattan J (2015). Revealing a cancer diagnosis to patients: attitudes of patients, families, friends, nurses, and physiccians in Lebanon-results of a crosssectional study. Current Oncology, 22(4): e264.

Girgis A, Sanson-Fisher RW (1995). Breaking bad news: consensus guidelines for medical practitioners. Journal of Clinical Oncology, 13(9), 2449-2456.

Grassi L, Giraldi T, Messina EG, Magnani K, Valle E, Cartei G (2000). Physicians' attitudes to and problems with truth-telling to cancer patients. Supportive Care in Cancer, 8(1), 40-45.

Hamadeh GN, Adib SM (1998). Cancer truth disclosure by Lebanese doctors. Social Science \& Medicine, 47(9), 1289-1294.

Hebert HD, Butera JN, Castillo J, Mega AE (2009). Are we training our fellows adequately in delivering bad news to patients? A survey of hematology/ oncology program directors. Journal of Palliative Medicine, 12(12), 11191124.

Holland JC, Weiss TR (2010). History of psycho-oncology. Psycho-oncology, 2, 3-12.

Kazdaglis GA, Arnaoutoglou C, Karypidis D, Memekidou G, Spanos G, Papadopoulos O (2010). Disclosing the truth to terminal cancer patients: a discussion of ethical and cultural issues. Eastern Mediterranean Health Journal, 16(4): 442.

Khalil RB (2013). Attitudes, beliefs and perceptions regarding truth disclosure of cancer-related information in the Middle East: a review. Palliative \& Supportive Care, 11(1), 69-78. 
Journal of Epidemiology and Public Health (2018), 4(2): 109-116

https://doi.org/10.26911/jepublichealth.2019.04.02.06

Law (2004). Patient rights and informed consent. J. Officiel Libanais June.

Mystakidou K, Parpa E, Tsilika E, Katsouda E, Vlahos L (2004). Cancer information disclosure in different cultural contexts. Supportive Care in Cancer, 12(3): 147-154.

Naji F, Hamadeh G, Hlais S, Adib S (2015). Truth disclosure to cancer patients: shifting attitudes and practices of Lebanese physicians. AJOB Empirical Bioethics, 6(3): 41-49.

Oksüzoğlu B, Abali H, Bakar M, Yildirim N, Zengin N (2006). Disclosure of cancer diagnosis to patients and their relatives in Turkey: views of accompanying persons and influential factors in reaching those views. Tumori, 92(1): 62-66.

Pugliese P, Perrone M, Nisi E, Garufi C, Giannarelli D, Bottomley A, Terzoli E (2006). An integrated psychological strategy for advanced colorectal cancer patients. Health and Quality of Life Outcomes, 4(1), 9.

Rosenbaum ME, Ferguson KJ, Lobas JG (2004). Teaching medical students and residents skills for delivering bad news: a review of strategies. Academic Medicine, 79(2), 107-117.

Surbone A (2006). Truth telling and ethical issues: an overview. In Proceeding of the UICC World Cancer Congress: 812.

Tsoussis S, Papadogiorgaki M, Markodimitraki E, Delibaltadakis G, Strevinas A, Psyllakis M, Krypotos S (2013). Disclosure of cancer diagnosis: the Greek experience. J BUON, 18(2), 516-526.

Wood WA, McCabe MS, Goldberg RM (2009). Commentary: disclosure in oncology-to whom does the truth belong?. The Oncologist, 14(1): 77-82. 